\title{
The Properties of Electromagnetic Light in Metals
}

\author{
Vahan Minasyan (Corresponding author) \\ Scientific Center of Applied Research, JINR, 141980, Russia \\ Tel: 7-496-216-2294Ｅ-mail: mvahan@scar.jinr.ru \\ Valentin Samoilov \\ Scientific Center of Applied Research, JINR, 141980, Russia \\ Tel: 7-496-216-5139Ｅ-mail: scar@off-serv.jinr.ru
}

\begin{abstract}
The recent years have seen a rapid expansion of research of the excitation of electromagnetic surface shape resonances in lamellar metallic gratings by light in the visible to near-infrared range based on the model of surface plasmon-polaritons. We argue that these electromagnetic waves propagate along metal-dielectric interfaces and can be guided by metallic nanostructures beyond the new fundamental Bose-particles with spin one and rest mass $m=2.5 \cdot 10^{-5} m_{e}$ (where $m_{e}$ is the mass of the electron). We call these light bosons because they induce the electromagnetic field. The existence of light bosons is confirmed by experiment connected with original type of the Bose-Einstein condensation. In this letter, we treat the interaction between light boson modes and electron modes in a metallic medium which leads to existence of the polaritons. These polaritons acquire a property of the charged electron gas with the Coulomb interaction and are excited into a fixed interval of wave numbers.
\end{abstract}

Keywords: Light particles with finite mass, Photons, Polaritons, Random-phase approximation

\section{Introduction}

There have been many studies of optical light transmission throughindividual nanometer-sized holes in opaque metal films in recent years, presented by Lopez-Rios et al. (1998), Ghaemi et al. (1998), and Sonnichen et al. (2000). These experiments showed highly unusual transmission properties of metal films perforated with a periodic array of subwavelength holes, because the intensity of electric field is highly localized inside the grooves (around 300-1000 times larger than intensity of incoming optical light. With the purpose of explaining these results, Minasyan \& Samoilov (2010) predicted the existence of light quasi-particles with spin 1 and finite effective mass $m=2.5 \cdot 10^{-5} m_{e}$, which may explain these experimental results. In this letter, we aim to change the concept of the predicted light quasi-particles because, as we argue, they represent fundamental neutral particles with finite mass $m$ and spin 1 .

As is well known, de Broglie (1925) first proposed the principle which states that a matter wave is determined by wave-particle duality or de Broglie wave of matter, which was confirmed by the famous Davisson and Germer experiment and also by the Compton effect where the particle nature of light was demonstrated. This reasoning helps us to treat the model of the electromagnetic field as a Bose gas consisting of Bose particles with spin 1 and non-zero rest mass which are interacting with each other due to gauge invariance. It is well known, the quantization scheme for the local electromagnetic field in vacuum was first treated by Planck in his black-body radiation studies. In this context, the classic Maxwell equations lead to appearance of the so-called ultraviolet catastrophe; to remove this problem, Planck modeled the electromagnetic field as an ideal Bose gas of massless photons with spin 1 . However, in this letter, we demonstrate that the non-ideal Bose gas of light particles represents as the Plank gas of massless photons.

The existence of light particles claims to consider their interaction with electron modes in a metallic medium. In this letter, we show that the given problem leads to existence of the polaritons with the energy spectrum expressed by the plasmon energetic gap. In fact, we prove that the polaritons acquire a property of a charged electron gas with the Coulomb interaction.

\section{Light particles with finite mass and spin 1}

We now investigate Maxwell's equations for a dielectric medium by quantum field theory: 


$$
\begin{gathered}
\text { curl } \vec{H}-\frac{1}{c} \frac{\partial \vec{E}}{\partial t}=0, \\
\operatorname{curl} \vec{E}+\frac{1}{c} \frac{\partial \vec{H}}{\partial t}=0, \\
\operatorname{div} \vec{E}=0, \\
\operatorname{div} \vec{H}=0,
\end{gathered}
$$

where $\vec{H}=\vec{H}(\vec{r}, t)$ and $\vec{E} \Rightarrow \vec{E}(\vec{r}, t)$ are, respectively, the vectors of local magnetic and electric fields depending on the coordinate $r$ and time $t ; c$ is the velocity of light in vacuum.

The Hamiltonian of the radiation field $\hat{H}_{R}$ is:

$$
\stackrel{ค}{H}_{R}=\frac{1}{8 \pi} \int\left(E^{2}+H^{2}\right) d V .
$$

In order to solve the problem connected with the quantized electromagnetic field in vacuum, we suggest that the transverse electromagnetic field in vacuum consists of light particles with spin 1 and rest mass $m$. Due to application of the principle of wave-particle duality, we can suggest that these particles have the vectors of the electric $\vec{E}_{0}=\vec{E}_{0}(r, t)$ and $\vec{H}_{0}=\vec{H}_{0}(\vec{r}, t)$, which satisfy the Maxwell equations owing to the application of the principle of gauge invariance:

$$
\begin{gathered}
\operatorname{curl} \vec{H}_{0}-\frac{1}{c} \frac{\partial \vec{E}_{0}}{\partial t}=0, \\
\operatorname{curl} \vec{E}_{0}+\frac{1}{c} \frac{\partial \vec{H}_{0}}{\partial t}=0, \\
\operatorname{div} \vec{E}_{0}=0, \\
\operatorname{div} \vec{H}_{0}=0 .
\end{gathered}
$$

To find the relationship between the vectors $\vec{E}=\vec{E}(\vec{r}, t), \vec{H}=\vec{H}(\vec{r}, t)$ and $\vec{E}_{0}=\vec{E}_{0}(\vec{r}, t)$ and $\vec{H}_{0}=\vec{H}_{0}(\vec{r}, t)$, we introduce the following expressions, which are in turn provided by the principle of gauge invariance:

$$
\vec{E}=\alpha \cdot \operatorname{curl} \vec{E}_{0}+\beta \vec{E}_{0}
$$

and

$$
\vec{H}=\alpha \cdot \operatorname{curl} \vec{H}_{0}+\beta \vec{H}_{0}
$$

where $\alpha$ and $\beta$ are unknown constants.

Obviously, Eqs (6)-(9) lead to following wave- equations:

$$
\nabla^{2} \vec{E}_{0}-\frac{1}{c^{2}} \frac{\partial^{2} \vec{E}_{0}}{\partial^{2} t}=0
$$

and

$$
\nabla^{2} \vec{H}_{0}-\frac{1}{c^{2}} \frac{\partial^{2} \vec{H}_{0}}{\partial^{2} t}=0
$$

Now, we can introduce the quantization form for the electric operator vector $\vec{E}_{0}$ of the light particles which is 
expressed via the second quantization vector wave functions of the light boson:

$$
\vec{E}_{0}=A\left(\varphi(\vec{r})+\varphi^{*}(\vec{r})\right)
$$

where $A$ is the amplitude of the electric field; $\varphi(\vec{r})$ and $\varphi^{*}(\vec{r})$ are, respectively, the second quantization wave vector functions for one light particle at the point of coordinate $r$ in the time $t$;

$$
\begin{gathered}
\varphi(\vec{r})=\frac{1}{\sqrt{V}} \sum_{\vec{k}} \vec{a}_{\vec{k}} e^{i(\vec{k} \vec{r}+k c t)} \\
\varphi^{*}(\vec{r})=\frac{1}{\sqrt{V}} \sum_{\vec{k}} \vec{a}_{\vec{k}}^{*} e^{-i(\vec{k} \vec{r}+k c t)}
\end{gathered}
$$

and

$$
\int \varphi^{*}(\vec{r}) \varphi^{*}(\vec{r}) d V=n_{0}+\sum_{\vec{k} \neq 9} \vec{a}_{k}^{*} \cdot \overrightarrow{a_{k}}=\hat{n}
$$

where $\vec{a}_{k}^{*}$ and $\overrightarrow{a_{k}}$ are, respectively, the Bose vector-operators of creation and annihilation for one free light particle with spin $1_{\rightarrow}$ described by the vector $k$,whose direction gives the direction of the electric field directed along unit,vector $e$ which is perpendicular to vector $k ; n$ is the operator total number of light bosons; $n_{0}=a_{0} a_{0}$ is the total number of light particles in the condensate.

Thus,

$$
\vec{E}_{0}=\vec{E}_{0,0}+\frac{A}{\sqrt{V}} \sum_{\vec{k} \neq 0}\left(\vec{a} \vec{k} e^{i(\vec{k} \vec{r}+k c t)}+\vec{a}_{\vec{k}}^{*} e^{-i(\vec{k} \vec{r}+k c t)}\right)
$$

Substitution of Eq. (18) into Eq. (7), by taking into account (13), leads to

$$
\vec{H}_{0}=\vec{H}_{0,0}-\frac{A}{\sqrt{V}} \sum_{\vec{k} \neq 0} \vec{k} \times\left(\frac{\vec{a} \vec{k}}{k} e^{i k c t}-\frac{\vec{a}_{-k}^{*}}{k} e^{-i k c t}\right) e^{i \vec{k} \vec{r}}
$$

While investigating superfluid liquid ${ }^{4} \mathrm{He}$, Bogoliubov (1947) separated the atoms of helium in the condensate from those atoms filling states above the condensate. In an analogous manner, we may consider the vector operators $\vec{a}_{0}$ and $\vec{a}_{0}^{*}$ as c-numbers $\vec{a}_{0}=\vec{a}_{0}^{*} \approx \vec{e} \sqrt{n_{0}}$ within the approximation of a macroscopic number of light particles in the condensate $n_{0} \gg>1$. This assumption leads to a broken Bose-symmetry law for light particles in the condensate. In fact, we may state that if $n_{0}$ number of light particles fill a condensate level with the wave vector $\vec{k}=0$, then they reproduce the electric vector $\overrightarrow{E_{0,0}}=2 A \sqrt{\frac{n}{V}} \vec{e}$ in Eq.(18), but in this case, the magnetic field of Light Particles into the condensate equals a zero because

$$
\vec{H}_{0,0}=-\frac{A}{\sqrt{V}} \cdot \lim _{\vec{k} \rightarrow 0} \frac{\vec{k}}{k} \times\left(\vec{a}_{0}-\vec{a}_{-0}^{*}\right) \approx-\frac{A}{\sqrt{V}} \cdot \lim _{\vec{k} \rightarrow 0} \frac{\vec{k}}{k} \times \vec{e}\left(\sqrt{n_{0}}-\sqrt{n_{0}}\right)=0
$$


This reasoning implies that light particles in the condensate may be physically observed by experiment.

With these new terms $\vec{E}_{0}$ and $\vec{H}_{0}$, by using Eqs.(6) and (7) in Eqs.(10) and (11), the radiation Hamiltonian $\stackrel{\cap}{H}_{R}$ (5) takes the form:

$$
\hat{H}_{R}=\frac{1}{8 \pi} \int\left(-\frac{\alpha}{c} \frac{\partial \vec{H}_{0}}{\partial t}+\beta \vec{E}_{0}\right)^{2} d V+\frac{1}{8 \pi} \int\left(\frac{\alpha}{c} \frac{\partial \vec{E}_{0}}{\partial t}+\beta \vec{H}_{0}\right)^{2} d V
$$

By prescription of Dirac, at the current time $t=0$, the operators $\frac{\partial \vec{E}_{0}}{\partial t}$ and $\frac{\partial \vec{H}_{0}}{\partial t} \quad$ take the forms:

$$
\frac{\partial \vec{E}_{0}}{\partial t}=\frac{i c A}{\sqrt{V}} \sum_{\vec{k}} k\left(\vec{a}_{\vec{k}}-\vec{a}_{-\vec{k}}^{*}\right) e^{i \vec{k} \vec{r}}
$$

and

$$
\frac{\partial \vec{H}_{0}}{\partial t}=-\frac{i c A}{\sqrt{V}} \sum_{\vec{k}}\left(\vec{k} \times \vec{a} \vec{k}-\vec{k} \times \vec{a}_{-k}^{*}\right) e^{i \vec{k}} \vec{r}
$$

In this respect, there is an important condition for the transverse electromagnetic field $\cdot \vec{E}_{0} \cdot \vec{H}_{0}=0$, which is easy to prove by using Eqs. (18) and (19), and the equality $\vec{a} \times \vec{b}=-\vec{b} \times \vec{a}$. Then, application of the equality

$$
\frac{1}{V} \int e^{i \vec{k} \vec{r}} d V=\delta(\vec{k})
$$

leads to the reduced form of $\stackrel{\cap}{H}_{R}$ :

$$
\stackrel{\cap}{H}_{R}=2 \sum_{k}\left(\frac{\hbar^{2} k^{2}}{2 m}+\frac{\beta^{2} A^{2}}{2}\right) \vec{a}_{\vec{k}}^{*} \cdot \vec{a} \vec{k}-\sum_{k}\left(\frac{\hbar^{2} k^{2}}{2 m}-\frac{\beta^{2} A^{2}}{2}\right)\left(\vec{a} \vec{k} \cdot \vec{a}-\vec{k}+\vec{a}_{-\vec{k}}^{*} \vec{a}_{\vec{k}}^{*}\right)
$$

if we suggest that $\alpha=\frac{\hbar \sqrt{2 \pi}}{\sqrt{m}}$.

We now apply a new transformation for the Minasyan \& Samoilov (2010) vector-operator, which is similar to the Bogoliubov (1947) one for the scalar operator, for evaluation of the energy levels of the operator $\stackrel{\hat{H}}{R}_{R}$ within the diagonal form:

$$
\vec{a}_{\vec{k}}=\frac{\vec{b}_{\vec{k}}+L_{\vec{k}} \vec{b}_{\vec{k}}^{*}}{\sqrt{1-L_{\vec{k}}^{2}}}
$$

where $L_{\vec{k}}$ is the real symmetrical functions of the wave vector $\vec{k}$.

The operator Hamiltonian $\stackrel{\hat{H}}{R}_{R}$, when using a canonical transformation in Eq.(24), takes the following form: 


$$
\stackrel{\cap}{H}_{R}=2 \sum_{\vec{k}} \varepsilon_{\vec{k}} \vec{d}_{\vec{k}}^{*} \cdot \vec{d}_{\vec{k}}
$$

Hence, we infer that the Bose operators $\vec{d}_{\vec{k}}^{*}$ and $\vec{d}_{\vec{k}}$ are, respectively, the vector creation and annihilation operators of free massless photons with the energy

$$
\varepsilon_{\vec{k}}=\sqrt{\left(\frac{\hbar^{2} k^{2}}{2 m}+\frac{\beta^{2} A^{2}}{2}\right)^{2}-\left(\frac{\hbar^{2} k^{2}}{2 m}-\frac{\beta^{2} A^{2}}{2}\right)^{2}}=\frac{\hbar k \beta A}{\sqrt{2 m \pi}}=\hbar k c
$$

where the constant $\beta=\frac{c \sqrt{2 \pi m}}{A}$.

Minasyan \& Samoilov (2010) found the boundary wave number $k_{0}=\frac{m c}{\hbar}$ for the electromagnetic field in vacuum, which appears by the suggestion that the light particles interact with each other by the repulsive potential $U_{k}$ in the momentum space:

$$
U_{k}=-\frac{\hbar^{2} k^{2}}{2 m}+\frac{m c^{2}}{2}>0
$$

As a result, the condition for the wave numbers of light particles $0 \leq k<k_{0}$ appears, providing, in its turn, the property of hard spheres for particles presented by Huang \& Yang (1957). Thus, there is a request of presence of a repulsive potential interaction between particles, which occurs namely for particles (recall S-wave repulsive pseudopotential interaction between atoms in superfluid liquid ${ }^{4} \mathrm{He}$ by the model hard spheres).

To find the mass of a light particle, we suggest that due to changing the energetic level in the hydrogen atom, the appearance of a photon with the energy $\hbar k c$ is determined by the distance between the energetic states for the electron going from a high level to a low one. The ionization energy of the hydrogen atom

$$
E_{d}=\frac{m_{e} e^{4}}{2 \hbar^{2}}
$$

is the maximal one for destruction of the atom, which in turn coincides with the maximal energy $\hbar k_{0} c$ in vacuum. Therefore, we have the important condition

$$
\frac{m_{e} e^{4}}{2 \hbar^{2}}=\hbar k_{0} c=m c^{2}
$$

which in turn determines the mass of the light particles $m=2.4 \cdot 10^{-35} \mathrm{~kg}$ in vacuum. Thus, we have proved that the light boson in vacuum is a fundamental particle because the rest mass $m$ is presented via fundamental constants. In this context, we may note that light bosons in vacuum are interacting moving particles which represent free massless photons. However, these particles can be observed as a constant electric field without a magnetic one, if they are in a condensate. This fact is confirmed by experiments connected with investigation of metal films because as Minasyan \& Samoilov (2010) argued the smooth metal-air interface should be regarded as a distinct dielectric medium, the skin of the metal, and found the boundary wave number

$$
k_{\varepsilon}=\frac{m c}{\hbar \varepsilon}
$$

for light particles of electromagnetic field in isotropic homogenous medium which treats Eq.(17) by following form:

$$
n_{0}+\sum_{0<k<k_{\varepsilon}} \vec{a}_{k}^{*} \cdot \overrightarrow{a_{k}}=\hat{n}
$$

where $\boldsymbol{\varepsilon}$ is the dielectric respond of metal's skin under an electromagnetic field in the visible to near-infrared 
range with frequency $\omega \leq \omega_{0}$ :

$$
\varepsilon=1+\frac{4 \pi N e^{2}}{m_{e}\left(\omega_{0}^{2}-\omega^{2}\right)}
$$

$N$ is the concentration of ions in metal's skin; $\omega_{0}$ is the resonance frequency.

There is observed a shape resonance in lamellar metallic gratings when frequency $\omega$ of optical light in the visible to near-infrared range coincides with resonance frequency of dipole $\omega_{0}$ in metal's skin. In this case, the boundary wave number tends to infinity:

$$
\lim _{\boldsymbol{w} \rightarrow \omega_{0}} k_{\varepsilon}=\lim _{\boldsymbol{w} \rightarrow \omega_{0}} \frac{m c}{\hbar \varepsilon}=\lim _{\boldsymbol{\omega} \rightarrow \omega_{0}} \frac{m c}{\hbar\left(1+\frac{4 \pi N e^{2}}{m_{e}\left(\omega_{0}^{2}-\omega^{2}\right)}\right)} \rightarrow 0
$$

which implies that all light particles fill the condensate level because total number $n_{0}$ of light particle into the condensate tends to $n$. There is observed the original type of the Bose condensation which does not depend on temperature but depends on the frequency of electromagnetic field. The intensity of electric field is highly localized inside the grooves around 300-1000. This fact is result of rising number of light particles into the condensate around $\sqrt{\frac{n}{n_{0}}}$ times, which may take around 10-30. In turn, the constant electric field in dielectric medium $\lim _{\boldsymbol{w} \rightarrow \omega_{0}} E_{0,0}=2 A \sqrt{\frac{n}{V}} \vec{e}$, presented in Eq.(18), (which in turn is not changing in the case of homogeneous dielectric medium,) is enhanced by $\sqrt{\frac{n}{n_{0}}}$ times. Therefore, the launching of surface electrons into nanoholes in metal films was observed in the condition of the resonance effect, which in turn, confirms the existence of light particles.

\section{Properties of the electromagnetic field in metals}

Now, we attempt to investigate the interaction of light particles with free electrons in a metal. To describe the properties of the model of a light boson gas and charged electron gas mixture confined in a box of volume $\mathrm{V}$, we present the Hamiltonian of a system which consists of the term of the Hamiltonian of the light boson gas $\stackrel{\cap}{H}_{R}$ and the term of the Hamiltonian of an ideal Fermi charged electron gas $\hat{H}_{e l}$ in addition to the term $\hat{H}_{Q}$ of the interaction between the density of the light boson modes and density of the charged electron modes which was first proposed by Dirac:

$$
\stackrel{\hat{H}}{=} \stackrel{\hat{H}}{R}_{R}+\stackrel{\cap}{H}_{Q}+\stackrel{\cap}{H}_{e l}
$$

In this context, the operator Hamiltonian of the free electron gas presents as:

$$
\stackrel{\cap}{H}_{e l}=\sum_{k, \sigma} \frac{\hbar^{2} k^{2}}{2 m_{e}}{\overrightarrow{b_{k, \sigma}^{*}}}^{*} \cdot \overrightarrow{b_{k, \sigma}},
$$

where $\overrightarrow{b_{k, \sigma}}$ and $\overrightarrow{b_{k, \sigma}}$ are, respectively, the Fermi operators of creation and annihilation for a free charged electron with the wave-vector $\vec{k} ; \sigma= \pm \frac{1}{2}$ is the value of the spin z-component.

To present the Hamiltonian $\hat{H}_{Q}$ of the interaction between light particle modes and charged electron modes, we introduce the method of second quantization for a system of $N$ fermions. In this respect, we may rewrite the second quantization wave functions for one electron at the point of the coordinate $\vec{r}$ as: 


$$
\begin{gathered}
\psi(r, \sigma)=\frac{1}{\sqrt{V}} \sum_{k, \sigma} \vec{b}_{k, \sigma} e^{i \vec{k} \cdot \vec{r}}, \\
\psi^{*}(\overrightarrow{r, \sigma})=\frac{1}{\sqrt{V}} \sum_{k, \sigma} \vec{b}_{k, \sigma}^{*} e^{-i \vec{k} \cdot \vec{r}}
\end{gathered}
$$

and

$$
\int \psi^{*}(\overrightarrow{r, \sigma}) \psi^{*}(\overrightarrow{r, \sigma}) d V=\sum_{k, \sigma} \overrightarrow{b_{k, \sigma}^{*}} \cdot \overrightarrow{b_{k, \sigma}}=N
$$

Now, we calculate the term $\stackrel{\hat{H}}{Q}_{Q}$ between light particle modes and charged electron modes:

$$
\hat{H}_{Q}=\sum_{\sigma} \int \psi^{*}(\overrightarrow{r, \sigma}) \hat{H}_{q} \psi^{*}(\overrightarrow{r, \sigma}) d V
$$

where $\hat{H}_{q}$ is the operator of the interaction between one electron and the electromagnetic field:

$$
\hat{H}_{q}=\frac{1}{2 m_{e}}\left[\left(\vec{p}-\frac{e \vec{A}}{c}\right)^{2}-p^{2}\right]
$$

where $\vec{p}=-i \hbar \nabla ; m_{e}$ and $e$ are, respectively, the mass and charge of the charged electron; $\vec{A}$ is the vector potential of the electromagnetic light.

Substituting Eq.(33) into Eq.(32) by using

$$
\frac{1}{V} \int e^{i \vec{k} \vec{r}} d V=\delta(\vec{k})
$$

and

$$
\vec{A}=\frac{1}{\sqrt{V}} \sum_{\vec{k}}\left(\vec{A} \vec{k} e^{i(\vec{k} \vec{r}+k c t)}+\vec{A}_{\vec{k}}^{*} e^{-i(\vec{k} \vec{r}+k c t)}\right),
$$

we obtain

$$
\begin{aligned}
& \hat{H}_{Q}=\frac{e^{2}}{2 m_{e} c^{2} V} \sum_{\vec{k}_{1}, \sigma} \sum_{\vec{k}_{2}, \sigma} \sum_{\vec{k}_{3}} \sum_{\vec{k}_{5}} \vec{b}_{k_{1}, \sigma}^{*} \vec{b}_{k_{2}, \sigma}\left(\vec{A}_{k_{3}}+\vec{A}_{-\vec{k}_{3}}^{*}\right)\left(\vec{A}_{\vec{k}_{4}}+\vec{A}_{-k_{4}}^{*}\right) \delta\left(\vec{k}_{2}+\vec{k}_{3}+\vec{k}_{4}-\vec{k}_{1}\right)+ \\
& +\frac{i e}{2 m_{e} c^{2} V} \sum_{\vec{k}_{1}, \sigma} \sum_{\vec{k}_{2}, \sigma} \sum_{\vec{k}_{3}} \vec{k}_{2} \cdot \vec{b}_{k_{1}, \sigma} \vec{b}_{k_{2}, \sigma}\left(\vec{A}_{\vec{k}_{3}}+\vec{A}_{-\vec{k}_{3}}^{*}\right) \delta\left(\vec{k}_{2}+\vec{k}_{3}-\vec{k}_{1}\right)- \\
& -\frac{i e}{2 m_{e} c^{2} V} \sum_{\vec{k}_{1}, \sigma} \sum_{\vec{k}_{2}, \sigma} \sum_{\vec{k}_{3}} \vec{k}_{3} \cdot \vec{b}_{k_{1}, \sigma} \vec{b}_{k_{2}, \sigma}\left(\vec{A}_{\vec{k}_{3}}+\vec{A}_{-\vec{k}_{3}}^{*}\right) \delta\left(\vec{k}_{2}+\vec{k}_{3}-\vec{k}_{1}\right),
\end{aligned}
$$

where $\vec{A} \vec{k}$ and $\vec{A}_{\vec{k}}^{*}$ are, respectively, the Bose vector-operators potential of the quantization electromagnetic field which are defined by "creation" and "annihilation" of light particles with the wave vector $\vec{k}$. 
To present $\stackrel{\cap}{H}_{Q}$ in the reduced form, it is applicable to use the random phase approximation (RPA), proposed by Bohm \& Pines (1953):

$$
\sum_{\substack{k_{1}, \sigma \\ b_{k}, \sigma}}^{b_{\overrightarrow{k_{1}}-k}^{*}} \underset{b_{\vec{k}}}{\rightarrow}=N \boldsymbol{\delta}(\vec{k})
$$

Then, the term of the operator $\hat{H}_{Q}$ takes the form:

$$
\stackrel{\cap}{H}_{Q}=\frac{e^{2} N}{2 m_{e} c^{2} V} \sum_{\vec{k}}\left(\vec{A}_{\vec{k}}+\vec{A}_{-\vec{k}}^{*}\right)\left(\vec{A}_{-k}+\vec{A}_{\vec{k}}^{*}\right) .
$$

To find the Bose operators $\vec{A} \vec{k}$ and $\vec{A}_{\vec{k}}^{*}$ via $\vec{a}_{\vec{k}}^{*}$ and $\vec{a}_{\vec{k}}$, we use the famous formula of electrodynamics

$$
\vec{E}=-\frac{\partial \vec{A}}{c \partial t}
$$

where $\vec{A}$ is presented by (34).

On the other hand, the vector electric field $\vec{E}$ may be presented as:

$$
\vec{E}=-\frac{\hbar \sqrt{2 \pi}}{c \sqrt{m}} \frac{\partial \vec{H}_{0}}{\partial t}+c \sqrt{2 \pi m} \vec{E}_{0},
$$

where $\vec{E}_{0}$ and $\vec{H}_{0}$ are presented by (12) and (13).

Comparing Eqs.(38) and (39) within application of Eqs. (34), (12) and (13), we obtain

$$
\vec{A} \vec{k}=-\frac{\hbar \sqrt{2 \pi}(\vec{k} \times \vec{a} \vec{k})}{k \sqrt{m}}+\frac{i c \sqrt{2 \pi m} \vec{a} \vec{k}}{k}
$$

and

$$
\vec{A}_{\vec{k}}^{*}=-\frac{\hbar \sqrt{2 \pi}\left(\vec{k} \times \vec{a} \overrightarrow{\vec{k}^{*}}\right)}{k \sqrt{m}}-\frac{i c \sqrt{2 \pi m} \vec{a}_{\vec{k}}^{*}}{k}
$$

Substituting the Bose operators $\vec{A} \vec{k}$ and $\vec{A}_{\vec{k}}^{*}$ from Eqs.(40) and (41) into Eq.(37), by using the conditions for the transverse electromagnetic wave, we may get to the approximated form of the operator $\mathrm{r} \hat{H}_{Q}$ :

$$
\stackrel{\cap}{H}_{Q}=-\sum_{\vec{k}} \frac{m e^{2} \pi N}{m_{e} k^{2} V}\left(\vec{a}_{\vec{k}}-\vec{a}_{-\vec{k}}^{*}\right)\left(\vec{a}_{-k}-\vec{a}_{\vec{k}}^{*}\right)-\frac{\hbar^{2} e^{2} \pi N}{m m_{e} c^{2} V} \sum_{\vec{k}}\left(\vec{a}_{\vec{k}}-\vec{a}_{-\vec{k}}^{*}\right)\left(\vec{a}_{-k}-\vec{a}_{\vec{k}}^{*}\right) .
$$

Inserting $\stackrel{\cap}{H}_{\underline{Q}}$ from Eq.(42) into Eq.(27) and using Eq.(23), we achieve the main part of the Hamiltonian of total 
radiation $\hat{H}_{R Q}$ :

$$
\begin{aligned}
& \hat{H}_{R Q}=\hat{H}_{R}+\hat{H}_{Q}=2 \sum_{k}\left(\frac{\hbar^{2} k^{2}}{2 m}+\frac{m c^{2}}{2}+\frac{m e^{2} \pi N}{m_{e} k^{2} V}+\frac{\hbar^{2} e^{2} \pi N}{m m_{e} c^{2} V}\right) \vec{a}_{\vec{k}}^{*} \cdot \vec{a} \vec{k}+ \\
& +\sum_{\vec{k}} U_{\vec{k}}\left(\vec{a}_{\vec{k}} \cdot \vec{a}_{-\vec{k}}+\vec{a}_{-\vec{k}}^{*} \vec{a}_{\vec{k}}^{*}\right)
\end{aligned}
$$

where

$$
U_{\vec{k}}=-\frac{\hbar^{2} k^{2}}{2 m}+\frac{m c^{2}}{2}-\frac{m e^{2} \pi \mathrm{N}}{m_{e} k^{2} V}-\frac{\hbar^{2} e^{2} \pi \mathrm{N}}{m m_{e} c^{2} V}
$$

is the repulsive potential between light particles, which must be positive within the above-mentioned model of hard spheres. Therefore, at $U_{\vec{k}}>0$ in Eq.(44), we have the inequality

$$
k^{4}-k^{2}\left(\frac{m^{2} c^{2}}{\hbar^{2}}-\frac{\Delta^{2}}{2 \hbar^{2} c^{2}}\right)+\frac{\Delta^{2} m^{2}}{2 \hbar^{4}}<0 .
$$

This inequality gives the solution $k_{2}<k<k_{1}$ where the boundary wave numbers are

$$
k_{1,2}=\frac{m c}{\sqrt{2} \hbar} \sqrt{1-\frac{\Delta^{2}}{2 m^{2} c^{4}} \pm \sqrt{\left(1-\frac{\Delta^{2}}{2 m^{2} c^{4}}\right)^{2}-\frac{2 \Delta^{2}}{m^{2} c^{4}}}},
$$

where $\Delta=e \hbar \sqrt{\frac{4 \pi N}{m_{e} V}}$ is the classical energetic plasmon gap presented by Bohm \& Pines (1953). For metals $m c^{2}>2 \Delta$ because $\Delta \approx 10^{-19} J$ and $m c^{2} \approx 2 \cdot 10^{-18} J$, therefore, we may accept the boundary wave vectors are

$$
k_{1} \approx \frac{m c}{\hbar}\left(1-\frac{\Delta^{2}}{2 m^{2} c^{4}}\right) ; \quad k_{2} \approx \frac{\Delta}{\sqrt{2} \hbar c},
$$

which in turn determine the wave numbers of the quantum state where light particles can exist.

We now apply a linear transformation of the vector Bose-operators Eq.(24) for evaluation of the energy levels of the operator $\hat{H}_{R Q}$ in (43) by diagonal forms. Thus,

$$
\hat{H}_{R Q}=2 \sum_{k_{2}<k<k_{1}} \chi_{\vec{k}} \vec{e}_{\vec{k}}^{*} \cdot e_{\vec{k}}
$$

Hence, we infer that the Bose-operators $\underset{\vec{k}}{\overrightarrow{e_{k}}}$ and $\underset{\vec{k}}{\vec{e}}$ are, respectively, the vector Bose-operators of the creation and annihilation of free polaritons with the energy

$$
\chi_{\vec{k}}=\sqrt{\hbar^{2} k^{2} c^{2}+\frac{\Delta^{2}}{2}+\frac{\Delta^{2} m^{2} c^{2}}{2 \hbar^{2} k^{2}}}
$$

because 


$$
\begin{aligned}
& \chi_{\vec{k}}^{2}=\left(\frac{\hbar^{2} k^{2}}{2 m}+\frac{m e^{2} \pi N}{m_{e} k^{2} V}+\frac{\hbar^{2} e^{2} \pi N}{m m_{e} c^{2} V}+\frac{m c^{2}}{2}\right)^{2}-\left(\frac{\hbar^{2} k^{2}}{2 m}+\frac{m e^{2} \pi N}{m_{e} k^{2} V}+\frac{\hbar^{2} e^{2} \pi N}{m m_{e} c^{2} V}-\frac{m c^{2}}{2}\right)^{2}= \\
& =4 \cdot \frac{m c^{2}}{2} \cdot\left(\frac{\hbar^{2} k^{2}}{2 m}+\frac{m e^{2} \pi N}{m_{e} k^{2} V}+\frac{\hbar^{2} e^{2} \pi N}{m m_{e} c^{2} V}\right)=\hbar^{2} k^{2} c^{2}+\frac{\Delta^{2} m^{2} c^{2}}{2 \hbar^{2} k^{2}}+\frac{\Delta^{2}}{2} .
\end{aligned}
$$

Obviously, the energy of polaritons is defined by the plasmon energetic gap $\Delta$ fixed in Eq.(47), which in turn determines the property of an electron gas with the Coulomb interaction. Indeed, the collective excitation of the density of the electron modes, due to application of the RPA, represents plasmon with the energy:

$$
E_{\vec{k}}=\sqrt{\frac{1}{3} \cdot \hbar^{2} k^{2} \overline{v^{2}}+\Delta^{2}}
$$

where $\overline{v^{2}}$ is the average square of the electron velocity which was introduced by Bohm \& Pines (1953). Hence, as wee see the Eqs.(47) and (48) contain the plasmon energetic gap $\Delta$ which reflects a property of electron gas with Coulomb interaction between electrons.

\section{Results}

In the present paper, the existence of a new fundamental particle is predicted in addition to the well-known electron, proton and neutron. Light particles with spin 1 and mass $m=2.4 \cdot 10^{-5} m_{e}$ can only be observed by experiment when they are in a condensate because they represent constant electric field. Indeed, as we have been seen in the section 2, the original type of the Bose condensation, which does not depend on temperature, is observed. This fact is result of rising of number light particles into the condensate which leads to the launching of surface electrons into nanoholes in metal films, at condition of the resonance effect. The experiments, presented by Lopez-Rios et al. (1998), Ghaemi et al. (1998), and Sonnichen et al. (2000), confirms the existence of light particles.

In this letter, we showed also that in a metallic medium, interaction between light particles with the electron modes of the electron gas induce the polaritons into the fixed interval of the wave numbers $k_{2}<k<k_{1}$. These polaritons acquire a property of charged electrons with the Coulomb interaction between electrons.

\section{References}

C.Sonnichen, A.C.Duch, G.Steininger, M.Koch, J.Feldman. (2000). Launching surface plasmons into nanoholes in metal films. Applied Physics Letters, 76, 140-142.

D. Bohm, D.Pines. (1953). Physical Review, 92, 609.

H.F.Ghaemi, D.E.Grupp, T.W.Ebbesen, H.J.Lezes. (1998). Surface plasmons enhance optical transmission through suwaveleght holes. Physical Review B, 58, 6779--6782I.

K.Huang, C.N.Yang. (1957). Physical Review, 105, 767.

L. de Broglie. (1925). Ann. Phys. (Paris) 3, 22.

N.N.Bogoliubov. (1947). On the theory of superfluidity. Journal of Physics (USSR), 11, 23.

P.A.M.Dirac. (1958). The principles of Quantum Mechanics. Clarendon press, Oxford.

T.Lopez-Rios, D.Mendoza, F.J.Garcia-Vidal, J.Sanchez-Dehesa, B.Panneter. (1998). Surface shape resonances in lamellar metallic gratings. Physical Review Letters, 81, 665-668.

V.N.Minasyan and V.N.Samoilov. (2010). Two New Type Surface Polaritons Excited into Nanoholes in Metal Films. Progress in Physics, 2, 3-6. [Online] Available: http://ptep-online.com/index_files/issues.html. 